\title{
Blunted Weight Loss at Incident Diabetes is a Strong Marker for Elevated Insulin Resistance in Type 2 Diabetes Mellitus
}

Navas Nadukkandiyil ${ }^{1 *}$, Chakrapani Mahabala², Hanadi Al Hamad ${ }^{1}$, Essa Al Sulaiti², Marwan Badri Ramadan ${ }^{1}$ and Noorudeen Kaladi kunnummal $^{2}$

${ }^{1}$ Kasturba Medical College; Mangalore; Manipal University, India

${ }^{2}$ Rumailah Hospital, Hamad Medical Corporation, Doha, Qata

\begin{abstract}
Aim: To find out the relation between insulin resistance and weight status along with other clinical markers in individuals who are newly detected with type 2 diabetes mellitus.

Materials and Methods: 50 patients recently detected with type 2 diabetes mellitus were recruited in this casecontrol study. Fasting blood glucose, fasting insulin, systolic blood pressure, Glycated Hemoglobin, fasting lipid profile, anthropometric measurements and physical examinations were noted. IR was determined by Homeostasis Model Assessment (HOMA-IR). Logistic regression was done to calculate the adjusted odds ratio for weight status, other clinical and biochemical markers which predict HOMA-IR value greater than or equal to 2.5 .

Results: The odds ratio for weight status was $44.338(p=0.022,95 \% \mathrm{Cl}: 1.743-1127.683)$. Odds ratio for Acanthosis Nigricans, shin spots, truncal obesity, lipid profile, HbA1c, BMI, waist-hip ratio were not significant. Difference in fasting blood sugar $(p=0.001)$, fasting insulin $(p<0.001)$, HOMA-IR $(p<0.001)$, BMI $(p=0.003)$ and waist hip ratio $(p<0.001)$ were statistically significant among those who did not have weight loss compared to those who had weight loss. Difference in lipid profile and urine microalbumin were not significant.
\end{abstract}

Conclusion: Our study showed that not losing weight at incident diabetes could be a marker of elevated insulin resistance.

Keywords: Blunted Weight loss; Insulin resistance; New onset Type 2 diabetes mellitus; HOMA2; IR Method

\section{Introduction}

The prevalence of cardiovascular complications is on the rise in type 2 diabetes mellitus. Sixty to $80 \%$ of people with diabetes die of cardiovascular complications [1]. Vascular changes that occurs in long standing diabetic patients is the major contributor to these late complications in diabetes [1]. It has been seen that the development of cardiovascular complications are not uniform among diabetic population [2]. Some patients remain free of these complications even after many years of treatment for diabetes.

Interestingly insulin resistance is the only component of the metabolic syndrome that has shown to be relatively constant in type 2 diabetes mellitus and predicts the long term cardiovascular outcomes at the onset of diabetes [3-5]. Several longitudinal and cross sectional studies have shown that insulin resistance and hyperinsulinemia are known to predict of cardiovascular complications in type 2 diabetes mellitus [6,7]. In addition to association, a moderate correlation has also been established between insulin resistance and severity of coronary artery disease (CAD) [8]. Hence it is important to identify insulin resistance at incident diabetes itself where risk prediction and stratification of individuals could be initiated at a much earlier stage.

Weight loss at the time of diabetes detection is common, but not losing the weight at incident diabetes could be a sign of insulin resistance. Earlier observation studies have shown that weight gain from the age of 20 years to middle age is associated with increased risk of hyperinsulinemia [9-11]. Insulin resistance and hyperinsulinemia is characterized by resistance to glucose uptake. In this phase there could be increased anabolic actions of insulin which may blunt the weight loss associated with onset of diabetes mellitus.

Hence the study was proposed to find out the relation between insulin resistance and weight status along with other clinical markers in individuals who are newly detected with type 2 diabetes mellitus.

\section{Materials and Methods}

A Case control study of 50 patients with newly detected type 2 diabetes mellitus were recruited in the study. Diabetes was defined according to the American Diabetes Association (ADA) definition, [12] and those full filling the ADA criteria were included in the study. Patients with known cases of thyrotoxicosis, hypothyroidism, liver cirrhosis, malignancies, HIV, tuberculosis, fever and diarrhoea more than one week of duration, patients with oedema were excluded from the study.

Individuals were divided into control and cases based on HOMAIR values. Individual were said to be insulin resistant if HOMA-IR value was 2.5 or above, which is considered to be the optimal cutoff value particularly with respect to the Indian population [13]. Twenty five patients whose HOMA-IR values less than 2.5 were defined as controls and 25 patients those with HOMA-IR values greater than or equal to 2.5 were defined as cases.

*Corresponding author: Navas Nadukkandiyil, Specialist, Hamad Medical Corporation, Geriatrics and Internal Medicine, Doha, Qatar 3050, Qatar, Tel: 0097433050426; E-mail: drnavasnk30@gmail.com

Received October 24, 2014; Accepted December 14, 2014; Published January 02, 2015

Citation: Nadukkandiyil N, Mahabala C, Hamad HA, Sulaiti EA, Ramadan MB et al. (2015) Blunted Weight Loss at Incident Diabetes is a Strong Marker for Elevated Insulin Resistance in Type 2 Diabetes Mellitus. J Diabetes Metab 6: 481 doi:10.4172/2155-6156.1000481

Copyright: () 2015 Nadukkandiyil N, et al. This is an open-access article distributed under the terms of the Creative Commons Attribution License, which permits unrestricted use, distribution, and reproduction in any medium, provided the original author and source are credited. 
Citation: Nadukkandiyil N, Mahabala C, Hamad HA, Sulaiti EA, Ramadan MB, et al. (2015) Blunted Weight Loss at Incident Diabetes is a Strong Marker for Elevated Insulin Resistance in Type 2 Diabetes Mellitus. J Diabetes Metab 6: 481. doi:10.4172/2155-6156.1000481

Page 2 of 3

Physical examination was carried out to look for the presence of Acanthosis Nigricans, shin spots, truncal obesity. Anthropometric measurements such has height, weight, waist circumference and hip circumference were noted as per world health organization (WHO) norms [14]. Body mass index (BMI) and waist-hip ratio was calculated. Weight loss was defined as loss weight more than $5 \%$ when compared to previous weight [15]. The change in weight whether the increase or decrease was noted based on recall by the patients at the time of diagnosis of diabetes. Self-reported weight status has been validated in previous studies [16].

Systolic blood pressure was recorded. Biochemical parameters such as fasting blood sugar, fasting lipid profile, urine microalbumin were measured using an auto analyzer Hitachi P800.

The coefficient of variation was $<2 \%$ and $<5 \%$ for intra and inter batch, respectively, in all cases. Glycated Hemoglobin (HbA1C) was measured by High Performance Liquid Chromatography (HPLC) using diamat automated glycosylated hemoglobin analyzer, Bio-Rad. Fasting Insulin levels were assayed using Insulin ELISA kit manufactured by DRG legal manufacturer Germany based on sandwich principle. The coefficient of variation was $<3 \%$ and $<5 \%$ of intra and inter batch assay respectively.

Insulin resistance was measured by Homeostasis model assessment HOMA 2 computerized method, [17] which has been shown to correlate well with the euglycemic clamp for use in epidemiological studies [18].

The study protocol was approved by the institutional ethics committee and written informed consent was obtained from study participants.

\section{Statistical analysis}

Independent sample $t$ test was done to compare means between two groups. Chi square test was done for categorical variables. Logistic regression was done to calculate the adjusted odds ratio for weight status, other clinical and biochemical markers which predict HOMAIR value greater than or equal to 2.5 . $\mathrm{P}<0.05$ was considered to be stastically significant. Analysis was done using Statistical Package for Social Sciences (IBM SPSS) version 20.

\section{Results}

Fifty patients with newly detected type 2 diabetes mellitus were analyzed in this case control study. 25 subjects had HOMA-IR value less than 2.5 (50\%) and 25 subjects had HOMA-IR value greater than $2.5(50 \%)$. Mean age of the study participants was $49.7 \pm 7.16$. Average $\mathrm{HbA1c}$, microalbumin, fasting blood sugar, fasting insulin and fasting lipid profile, systolic blood pressure, BMI and waist-hip ratio in cases and controls are shown in Table 1. Blunting of weight loss at incident diabetes is defined as weight loss less than $5 \%$ is associated with increased insulin resistance. Twenty (80\%) subjects with HOMA-IR values greater than $2.5 \mathrm{did}$ not have weight loss compared to five $(20 \%)$ among subjects with HOMA-IR values less than $2.5(\mathrm{p}<0.001)$. The adjusted odds ratio for weight status was 44.338 ( $\mathrm{p}=0.022$, 95\%CI: 1.743 - 1127.683). The adjusted odds ratio for Acanthosis Nigricans, shin spots, truncal obesity, lipid profile, $\mathrm{HbAlc}$, BMI, waist-hip ratio was not significant (Table 2). Difference in fating blood sugar $(\mathrm{p}=0.001)$, fating insulin $(\mathrm{p}<0.001)$, HOMA-IR $(\mathrm{p}<0.001)$, BMI $(\mathrm{p}=0.003)$ and waist hip ratio $(\mathrm{p}<0.001)$ were statistically significant among those who did not have weight loss compared to those who had weight loss (Table
3). Difference in lipid profile and urine microalbuminuria were not significant (Table 3 ).

\section{Discussion}

In this case control study, we compared the weight status and other clinical and biochemical markers to predict the insulin resistance state in patients with newly detected treatment naïve type 2 diabetes mellitus. Patients who has not lost weight at incident diabetes are 44 times more likely to have HOMA-IR value of 2.5 and above when compared to those who had lost weight.

\begin{tabular}{|c|c|c|c|}
\hline Variable & HOMA-IR $<\mathbf{2 . 5}$ & HOMA-IR $\geq \mathbf{2 . 5}$ & P value \\
\hline Fasting blood sugar (mg/dl) & 179.28 & 222 & 0.007 \\
\hline Insulin $(\boldsymbol{\mu l U / m l )}$ & 10.82 & 27.89 & $<0.001$ \\
\hline HbA1C & 9.76 & 10.17 & 0.445 \\
\hline Triglycerides (mg/dl) & 166.72 & 163.92 & 0.81 \\
\hline HDL-C (mg/dl) & 37.72 & 35.08 & 0.238 \\
\hline LDL-C (mg/dl) & 125.16 & 129 & 0.673 \\
\hline VLDL-C (mg/dl) & 34.72 & 34.49 & 0.929 \\
\hline Microalbuminuria (mg/l) & 0.52 & 0.56 & 0.782 \\
\hline BMI & 22.04 & 24.36 & 0.015 \\
\hline Waist-Hip ratio & 0.914 & 0.959 & 0.029 \\
\hline Systolic Blood Pressure (mmHg) & 134.8 & 140 & 0.235 \\
\hline
\end{tabular}

Table 1: Mean clinical and biochemical parameters in subjects with HOMA -IR < 2.5 and HOMA-IR $>2.5$.

\begin{tabular}{|c|c|c|c|c|}
\hline Variable & Wald & p value & Odds Ratio & $\begin{array}{c}95 \% \text { Cl for Odds } \\
\text { Ratio }\end{array}$ \\
\hline Weight Status & 5.274 & 0.022 & 44.338 & $1.74-1127.68$ \\
\hline Acanthosis Nigricans & 0.008 & 0.928 & 0.887 & $0.066-11.900$ \\
\hline Shin Spot & 1.206 & 0.272 & 0.146 & $0.005-4.518$ \\
\hline Scleroderma & 1.322 & 0.25 & 0.192 & $0.011-3.204$ \\
\hline Osmotic & 1.598 & 0.206 & 0.195 & $0.015-2.459$ \\
\hline Truncal Obesity & 0.006 & 0.938 & 0.922 & $0.119-7.124$ \\
\hline BMI & 1.373 & 0.241 & 0.286 & $0.035-2.319$ \\
\hline Triglycerides & 1.336 & 0.248 & 0.981 & $0.949-1.014$ \\
\hline LDL-C & 0.149 & 0.699 & 1.008 & $0.966-1.053$ \\
\hline VLDL-C & 2.261 & 0.133 & 1.113 & $0.968-1.279$ \\
\hline Waist-hip ratio & 0.277 & 0.599 & 0.003 & $0.000-$ \\
\hline Microalbuminuria & 0.002 & 0.965 & 0.946 & $0.081-11.104$ \\
\hline HbA1C & 0.473 & 0.492 & 0.755 & $0.338-1.684$ \\
\hline Age & 0.91 & 0.34 & 0.885 & $0.688-1.138$ \\
\hline Sex & 2.41 & 0.121 & 0.121 & $0.008-1.741$ \\
\hline
\end{tabular}

Table 2: Odds ratio to predict HOMA-IR $\geq 2.5$.

\begin{tabular}{|c|c|c|c|}
\hline Variable & $\begin{array}{c}\text { Weight loss at } \\
\text { onset of Diabetes }\end{array}$ & $\begin{array}{c}\text { No weight loss at } \\
\text { onset of Diabetes }\end{array}$ & p value \\
\hline Fasting Blood sugar & 174.88 & 226.4 & 0.001 \\
\hline Insulin & 11.65 & 27.07 & $<0.001$ \\
\hline HOMA-IR & 1.76 & 4.52 & $<0.001$ \\
\hline BMI & 21.81 & 24.6 & 0.003 \\
\hline Waist-hip ratio & 0.88 & 0.98 & $<0.001$ \\
\hline Triglycerides & 162.02 & 168.56 & 0.576 \\
\hline LDL-C & 126.56 & 127.6 & 0.909 \\
\hline VLDL-C & 36 & 33.22 & 0.291 \\
\hline HbA1c & 9.73 & 10.2 & 0.385 \\
\hline Microalbumin & 0.44 & 0.64 & 0.162 \\
\hline
\end{tabular}

Table 3: Biochemical and anthropometric differences in patients with and without weight loss at the onset of diabetes. 
Citation: Nadukkandiyil N, Mahabala C, Hamad HA, Sulaiti EA, Ramadan MB, et al. (2015) Blunted Weight Loss at Incident Diabetes is a Strong Marker for Elevated Insulin Resistance in Type 2 Diabetes Mellitus. J Diabetes Metab 6: 481. doi:10.4172/2155-6156.1000481

Page 3 of 3

Other parameters did not reach statistical significant after adjusting for weight status. Hence not losing weight at the time of diabetes could be a marker for significantly elevated insulin resistance.

Those without weight loss have high blood sugar, insulin, HOMAIR, BMI, waist-hip ratio, truncal obesity and yet are less symptomatic when compared to those with weight loss. Hence diagnosis could be delayed and higher potential for undetected complications in those with blunted weight loss and higher insulin resistance. The hyperlipidemia and other clinical status were not different between these two groups. This observation highlights that the pathophysiology of insulin resistance could be different between these groups.

Diabetes itself is a heterogeneous disease, the profile and complications could be different among diabetic population [2]. Most of long term diabetes complications are vascular in nature.

Insulin resistance has shown to be associated with endothelial dysfunction and has a central role in atherosclerosis and is closely associated with increased cardiovascular disease $[19,20]$.

Studies have been reported where long term cardiovascular complications could be predicted by the measure of insulin resistance $[5,6]$. Since insulin resistance is constant in the diabetic population, its unique evolution will play a critical role pathogenesis of coronary artery disease.

Thus there is a need to identify insulin resistance in the early part of diabetes. The other clinical markers of metabolic syndrome like truncal obesity and BMI has low specificity and sensitivity in identifying IR, but in our study we observed that not losing weight at the time of diabetes could be a reliable marker for elevated insulin resistance.

Biological explanation for this observation could be hyperinsulinemia as an underlying feature for weight gain. In diabetes, insulin resistance is manifested by decreased insulin stimulated glucose transport and impaired suppression of hepatic glucose output, these functional defects are as a result of impaired insulin signaling in target issues and down regulation of the major insulin mediated glucose transporter, GLUT 4 which results in elevated blood glucose and insulin levels. Insulin being an anabolic hormone mediates its response through different receptors leading to increased accumulation of abdominal fat which further lead to increase in body weight.

Patients with Type 2 diabetes are from a heterogeneous population of risk profiles. Physicians and researchers should stratify these patients into different risk categories. Weight loss at the incident diabetes is common but not losing weight at the time of diagnosis of diabetes could be one of the important marker to predict elevated insulin resistance in the individuals.

Individuals not losing weight at the time of diagnosis of diabetes more likely to have high insulin resistance when compared to those who lose weight. Hence the treatment between these two groups should be different. Thus lifestyle modification should be much more intensive and metformin should be initiated in full dose in those who are not losing weight at the onset of diabetes to lower the insulin resistance which would in turn minimize the cardiovascular complications in this phenotype.

This study has few strengths and limitation. Strength would be, the weight status was recorded at the incident diabetes. Since weight change in the same individual in taking into consideration, inter individual variations have been neutralized. Limitation of the study is its small sample size.
In future longitudinal studies should be carried out on serial weight change and insulin resistance in healthy and diabetic individuals.

\section{Conclusion}

Our study showed that blunted weight loss at the time of diagnosis of diabetes is a strong and reliable indicator of elevated insulin resistance and these individuals should be managed aggressively for optimal cardiovascular outcomes.

\section{References}

1. Campbell NRC, Leiter L, Larochelle P, Tobe S, Chockalingam A, et al. (2009) Hypertension in diabetes: a call to action. The Canadian Journal of Cardiology 25: 299-302.

2. Tuomi T, Santoro N, Caprio S, Cai M, Weng J, et al. (2013) The many faces of diabetes: a disease with increasing heterogeneity. Lancet 6736: 1-11.

3. Kendall DM, Cuddihy RM, Bergenstal RM (2009) Clinical application of incretinbased therapy: therapeutic potential, patient selection and clinical use. The American journal of medicine 122: S37-50

4. Matthews DR, Cull CA, Stratton IM, Holman RR, Turner RC (1998) UKPDS 26 : Sulphonylurea failure in non-insulin-dependent diabetic patients over six years. UK Prospective Diabetes Study (UKPDS) Group. Diabetic medicine: a journal of the British Diabetic Association 15: 297-303.

5. Reaven GM (1988) Banting lecture 1988. Role of insulin resistance in human disease. Diabetes 37: 1595-1607.

6. Bonora E, Kiechl S, Willeit J, Oberhollenzer F, Egger G, et al. (2007) Insulin resistance as estimated by homeostasis model assessment predicts incident symptomatic cardiovascular disease in caucasian subjects from the general population: the Bruneck study. Diabetes care 30: 318-324.

7. Verhagen SN, Wassink AM, van der Graaf Y, Gorter PM, Visseren FL (2011) Insulin resistance increases the occurrence of new cardiovascular events in patients with manifest arterial disease without known diabetes. The SMART study. Cardiovascular diabetology 10: 100.

8. Mahabala C, Srinivasan M, Manjrekar P, Unnikrishnan B, Ullal A, et al. (2013) Correlation of severity of coronary artery disease with insulin resistance. North American Journal of Medical Sciences 5: 611.

9. Everson SA, Goldberg DE, Helmrich SP, Lakka TA, Lynch JW, et al. (1998) Weight gain and the risk of developing insulin resistance syndrome. Diabetes Care 21: 1637-1643.

10. Lakka HM, Salonen JT, Tuomilehto J, Kaplan GA, Lakka TA (2002) Obesity and weight gain are associated with increased incidence of hyperinsulinemia in non-diabetic men. Hormone and metabolic research 34: 492-498.

11. Chang Y, Sung E, Yun KE, Jung H, Kim C, et al. (2013) Weight Change as a Predictor of Incidence and Remission of Insulin Resistance. Plos One 8

12. Diabetes DOF (2010) Diagnosis and classification of diabetes mellitus. Diabetes care 33: S62-69.

13. Singh Y, Garg MK, Tandon N, Marwaha RK (2013) A Study of Insulin Resistance by HOMA-IR and its Urban Indian Adolescents. J Clin Res Pediatr Endocrinol 5: 245-251.

14. WHO (1995) Physical status: The use and interpretation of anthropometry Geneva: Report of a WHO expert Committee, WHO technical report series 854: 324.

15. Bistrian B (2012) Nutritional Assessment. Goldman's Cecil Medicine (24thedtn) Elsevier; Philadelphia, USA.

16. Christian NJ, King WC, Yanovski SZ, Courcoulas AP, Belle SH (2013) Validity of self-reported weights following bariatric surgery. JAMA 310: 2454-2456

17. Wallace Tm, Levy JC, Matthews DR (2004) Use and abuse of HOMA modelling Diabetes care 27: 1487-95

18. Nishio K, Fukui T, Tsunoda F, Kawamura K, Itoh S, et al. (2005) Insulin resistance as a predictor for restenosis after coronary stenting. Int $\mathrm{J}$ Cardiol 103: $128-134$.

19. Zornitzki T, Ayzenberg O, Gandelman G, Vered S, Yaskil E, et al. (2007) Diabetes, but not the metabolic syndrome, predicts the severity and extent of coronary artery disease in women. QJM 100: 575-581.

20. Aziz A, Wheatcroft S (2011) Insulin resistance in Type 2 diabetes and obesity: Implications for endothelial function. Expert Rev Cardiovasc Ther 9: 403-407. 